\title{
PROBLEM OF THE OBJECTS DETECTION ON LOW QUALITY 3D IMAGE - EXAMPLE SOLUTION
}

\begin{abstract}
Precise detection of analyzed objects is crucial and the most important task in quantitative and qualitative image of the microstructure analysis. The most common image distortion is noise, artefacts and low contrast. In the paper, author on the example of poor quality image of the polyurethane-polystyren composite structure, obtained with the X-ray microtomograph, presented possible methods of noise reduction, contrast enhancement, and correction of detection errors on the binary image.
\end{abstract}

Keywords: image processing, low contrast, noise, X-ray microtomography

\section{Introduction}

Analysis of the material structures using computer image analysis and stereology techniques is a standards in everyday practice in research and quality control laboratories. Number of professional software's, with build in automatic procedures for detection and quantitative analysis made the analysis fast and easy. No particular knowledge of image processing or stereology for standard analysis of good quality images is required from the operator of the analysis system. However, there are issues in which automatic procedures are powerless. Some materials due to its characteristic specific are difficult to imaging, like presented in the article composite. Analysis of spatial arrangement of the particles is possible to carry out on 3D images from X-ray micro tomography or on the set of the 2D images of cross sections of the sample using stereological methods. As can be observed on fig. 1, the problem with detection on 2D images is caused by semi-transparent matrix, which make visible the particles of polystyrene below the surface, and its detection and analysis may effect on the accuracy of the analysis. Imaging $3 \mathrm{D}$ by $\mathrm{X}$-ray micro tomography technique allows to obtained view inside the material structure without destroying the sample. Advantages of the 3D imaging were appreciated and nowadays this method is wildly applied in material science (SALVo L. et al. 2003; WANG L.B. et al. 2004, LANDIS E.N., KEANE D.T. 2010). However its limitations, like dependence the contrast of imaging objects from atomic number of chemical elements of tested materials, or spatial resolution on size of analysed sample may reduce the area of 3D imaging by X-ray micro tomography application (SONKA M. et al. 2014).

\footnotetext{
${ }^{1}$ Phd., Cracow University of Technology, Institute of Applied Informatics, gadek@ mech.pk.edu.pl
} 
Some image imperfections may be reduced by image processing techniques. In the paper author will present how combination of filtering, arithmetic and morphological transformation allow to obtain the correct object detection, and its further quantitative description by set of stereological parameters (GĄDEK A., WOJNAR L. 2003, RUSS J.C. 2011, GĄDEK-MOSZCZAK A., ŻMUDKA S. 2013).

\section{Research material}

3D analysis was done for the composite material polyurethane - polystyrene. A sample of dimensions: $62.5 \mathrm{~mm}$ x $69 \mathrm{~mm}$ x $62 \mathrm{~mm}$ was tested (Fig. 1).

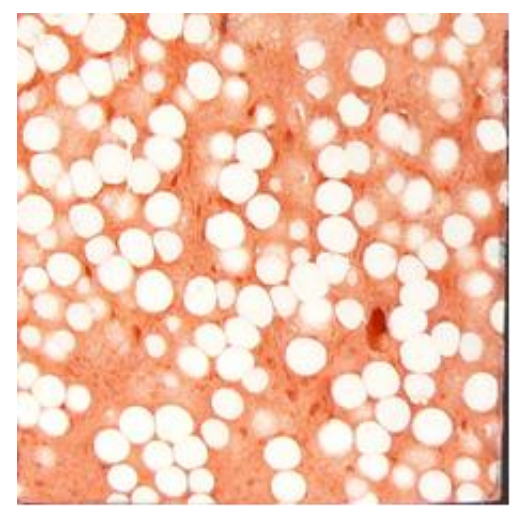

Fig. 1. Photography of the composite's sample cross section.

Source: own study

The three-dimensional image of the structure of the material under investigation was obtained using the Nanotom S high-resolution nanotomomograph scanner from General Electric.

As a result of the acquisition, a 3-D sample image of $1044 \times 1152 \times 1044$, with a resolution of $1 \mathrm{px}=0.06 \mathrm{~mm}$, was recorded in grayscale, with a 32 bit depth in a lossless RAW format. Reconstruction of the 3D image reveal the low quality of image due to low difference in $\mathrm{x}$-ray absorption. If the components of the composite consist of chemical elements with similar atomic number, X-ray microtomgraphy results in images with low contrast between them, what makes detection difficult and in some cases even impossible. And in those cases developing dedicated image processing algorithms for image quality improvement may allow to carry out analysis. 


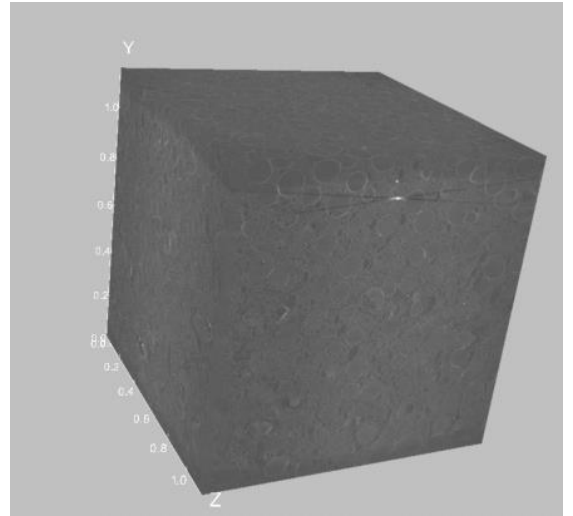

Fig. $2.3 D$ reconstruction of the $X$-ray microtomography image.

Source: own study

Visual assessment of exemplary cross section X-ray microtomography image of tested composite with polyurethane matrix and polystyrene filer allows to conclude that the noise level is relatively high and contrast between polystyrene image and polyurethane is very low (Fig. 2, 3a), what was confirmed by measured profile (Fig. $3 a)$. Actually the values of the voxels representing polyurethane and polystyrene are very similar. The space between the edge of the polystyrene and polyurethane gives the lightest voxels on the image.

An attempt to detect the polystyrene ball without any correction, on raw image by binarization led to failed results (Fig. 3b, 3c). On Fig. 3b the range of voxels value was to wide, and many artefacts were as an object detected. Narrowing the range of voxels values (115-203) resulted in binary image of discontinuous edges, and artefact as well.

Additionally the problem with thresholds level setting is illustrate by the profile curve run. The range of values representing analysing structure is narrow, between the 80 and 140. The range of image is (0.203), but extreme values of the interval appear incidentally, and they have no relation to the individual elements of the structure. Slightly change of threshold levels effects (Fig. 3b, 3c) results in significant change in detection result.

Failed attempts of polystyrene ball detection on the raw image have confirmed that it is necessary to perform image processing to improve its quality. 

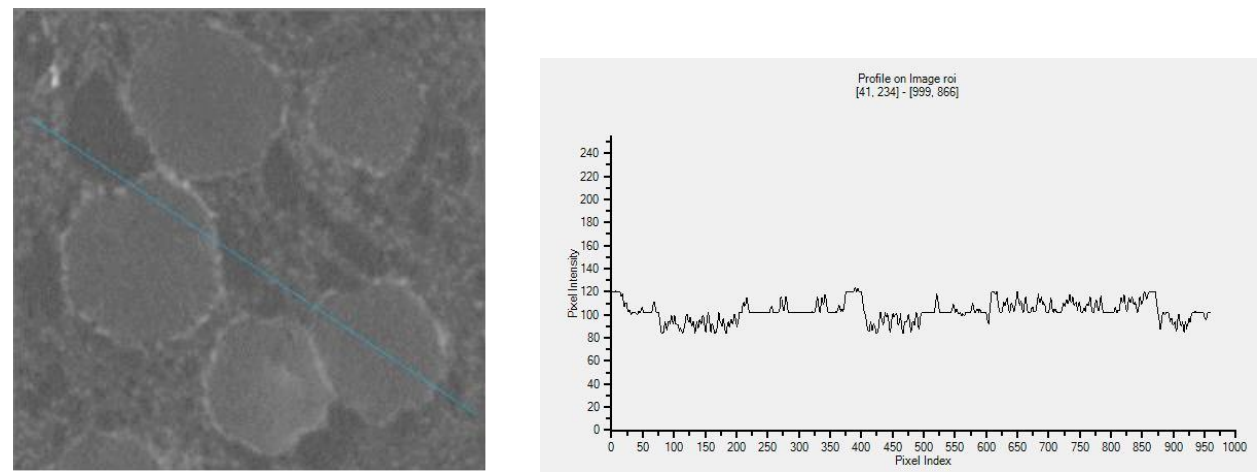

a)

Selected frame of cross section image and its profile

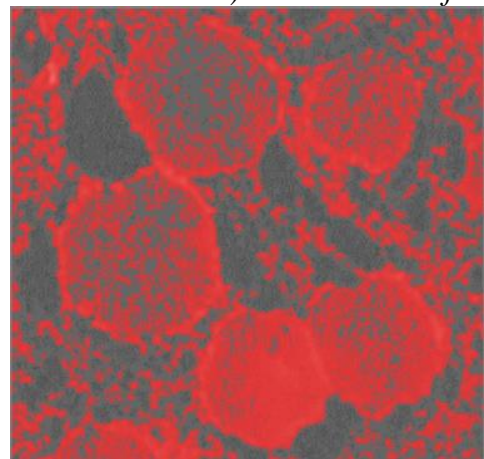

b) Result of detection test with threshold 103-203

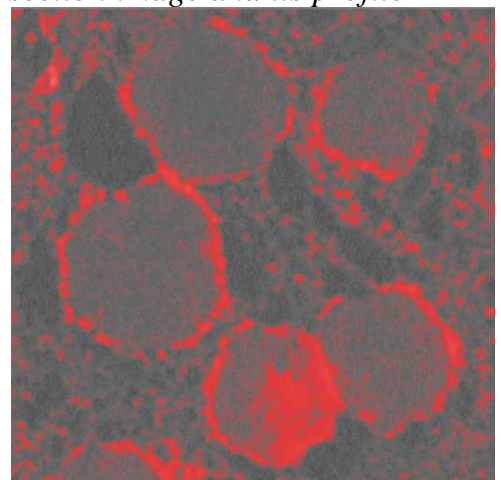

c) Result of detection test with threshold 115-203

Fig. 3. Analysis of t image defects and their impact on detection.

Source: own study

\section{Image processing}

Image processing and quantitative evaluation were performed in Aphelion 4.1. ADCIS company. Aphelion is a professional software for image processing and analysis which allows researcher to develop automatic algorithms dedicated to analysed images 2 and 3 dimensional.

In order to reduce noise and homogenize the image areas corresponding to polystyrene beads, median filtration was performed. Median filtration is very effective tool for noise reduction and simultaneously keeps sharp edges of objects (RUSS J.C. 2011). Afterwards obtained image is transformed by filter indicating local variance of image matrix, resulting in an image with very strongly contrasted edges of the objects. Such an image is subjected to a mathematical morphology transformations, first close operation by performing a dilatation and then on a resulting erosion. These transformations have caused the objects edge lines to merge. 
On such prepared image the edge detection by binarization transformation was perform. As a result of binarization, binary image were obtained, where the edges correspond to the value of 1 and the remaining points of the image (background) assume a value 0 (Fig. 4).

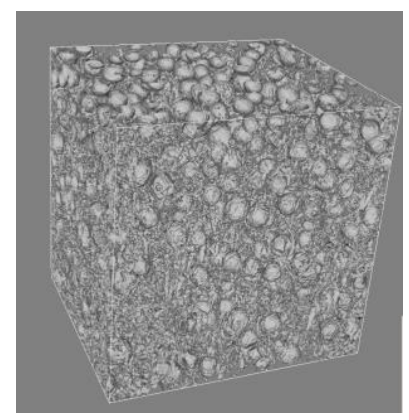

Fig. 4. 3D reconstruction of the binary image of the sample being tested.

Source: own study

After binarization the images of the edges of objects were obtained. To represent the objects and quantitatively described them, the further morphological transformation is needed. Firstly the filling the area inside the objects. Aphelion allow to perform this transformation using build in algorithm. This algorithm analyses the image and fills the discontinuity area within the closed edges with values 1 .

Then, to separate the interconnected objects, we perform a separation transformation, based on the watershed segmentation on Euclidian distance function image.

On the binary images these are artefacts, volume corresponding to pores in the material of matrix or unreduced noise. In order to perform an automated quantitative polystyrene analysis, we must remove all other objects from the image.

The first step in the filtration process is to perform the opening morphological transformation but limited only to the small objects, defined by volume voxels, which are with high probability the effect of the noise. This is a transformation by which all objects that meet the established size criterion are removed from the image. In our case, all objects up to 100000 voxels have been removed. The effect of the filtration performed can be seen in Fig. 5.

Next stage of the processing preparing image to automatic measurements is clustering. Clustering is the transformation which analyse whole image searching for continuous volume of voxels - voxel clusters of value 1 . 


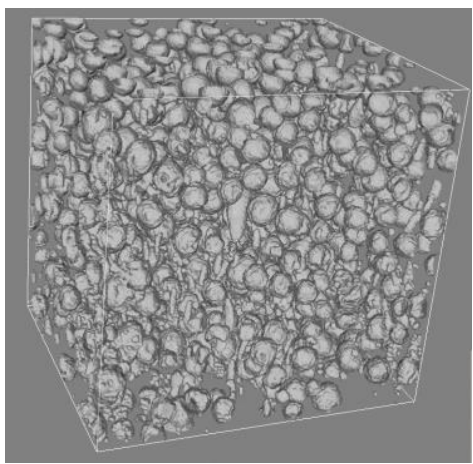

Fig. 5. Image after the limited opening transformation.

Source: own study

Each detected cluster receives a sequence number (identification label). Only images after clustering can be quantified using automated algorithms.

The clustering effect can be visualized by giving each object a randomly selected backlight colour. Thanks to this treatment, visual assessment of the effects of our corrective actions is much easier (Fig. 6a).

The last action before starting the measurement algorithms is to remove all objects cut across the edges of the image. As a result of this action we get the image presented in Figure 6, which is the final image at the stage of preparation for quantitative analysis. The next and final activity is quantitative analysis, which will allow quantitative objects.

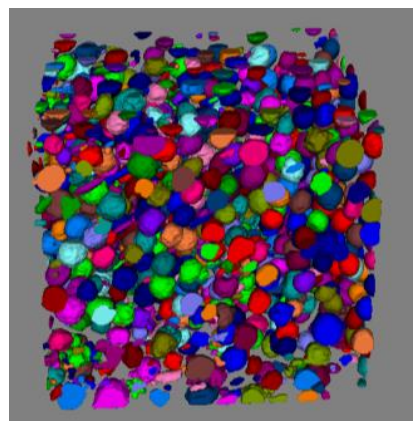

a)

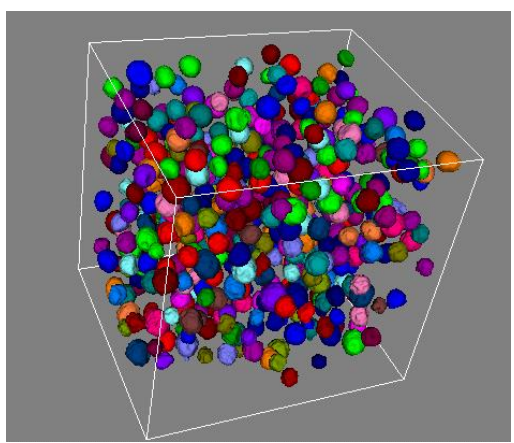

b)

Fig. 6. a) Image after clustering, b) final image.

Source: own study 


\section{Discussion and conclusion}

Correctly, exactly detected objects on image allow for precise quantitative analysis based on numerous stereological parameters indication, like volume fraction, number of objects per volume unit, mean volume, shape analysis or analysis of special arrangement of the particles. Image processing allow to reduce the effect of the image distortion, but how it can be observed on fig. 4 and 5, it is extremely difficult to remove it completely. It must be also taken into account that all image processing by transforming image, introduce on image new values, and change the original information which were an effect of acquisition process. Some mage transformation may even cause its partial loss. If there is possible to verify obtained results, by other method, it should be done.

It is well know that images for automatic quantitative analysis need to characterized high quality. Human perception is much more efficient in recognizing objects even in poor quality images. It is caused by different way of analysis images. The human mind analyses the image multithreaded, contextually, locally and globally. Finds patterns and matches them to the image, still learning. Comparing to that all working out image processing algorithms seems to be very primitive.

But despite it limitation, as it is shown, combination of transformation and filtration may lead out to success detection.

The images are full of information about the materials being studied, which may have essential effect on its mechanical, physical properties. The structure of analysed materials may derive important information about manufacturing technology, and it is well worth the effort to develop a dedicated method for processing and detecting the object.

\section{Bibliography}

1. GądeK-MoszcZak A., ŻMUdKa S. 2013. Description of 3D microstructure of the composites with polypropylene (PP) matrix and tuf particles fillers. Solid State Phenomena 197, 186-191

2. GĄDEK A., WoJNAR L., 2003. Wptyw sposobu korekcji cienia i doboru metody detekcji na wyniki ilościowej oceny porowatości. Inżynieria Materiałowa, 24 (3), 111-116

3. LANDIS E.N., KEANE D.T. 2010. X-ray microtomography, Materials Characterization, Vol. 61 , issue $12,1305-1316$

4. Russ J.C. 2011. The Image Processing Handbook, Sixth Edition, CRC Press, Inc. Boca Raton, FL, USA

5. Salvo L., Cloetens P., Maire E., Zabler S, Blandin J.J, Buffière, Ludwig W., Boller E, Bellet D., JosSEROND C. 2003. X-ray micro-tomography an attractive -140 - 
characterisation technique in materials science. Nuclear Instruments and Methods in Physics Research Section B: Beam Interactions with Materials and Atoms; 200 (2003) 273-286.

6. SONKa M., HLAVAC V., BoYLE R. 2014. Image processing, analysis, and machine vision. Cengage Learning, Stamford, USA

7. Wang L. B., Frost J. D., AND LAI J. S. 2004. Three-Dimensional Digital Representation of Granular Material Microstructure from X-Ray Tomography Imaging. Journal of Computing in Civil Engineering, Vol. 18, Issue 1. DOI: http://dx.doi.org/10.1061/(ASCE)CP.1943-5487.0000270 